Research Article

\title{
Connection-Based Multiplicative Zagreb Indices of Dendrimer Nanostars
}

\author{
Aqsa Sattar, ${ }^{1}$ Muhammad Javaid $\left(\mathbb{D},{ }^{1}\right.$ and Ebenezer Bonyah $\mathbb{1 D}^{2}$ \\ ${ }^{1}$ Department of Mathematics, School of Science, University of Management and Technology (UMT), Lahore 54770, Pakistan \\ ${ }^{2}$ Department of Mathematics Education, Akenten Appiah-Menka University of Skills Training and Enterpreneurial Development, \\ Kumasi 00233, Ghana
}

Correspondence should be addressed to Ebenezer Bonyah; ebbonya@gmail.com

Received 18 November 2021; Accepted 8 December 2021; Published 23 December 2021

Academic Editor: Gohar Ali

Copyright (C) 2021 Aqsa Sattar et al. This is an open access article distributed under the Creative Commons Attribution License, which permits unrestricted use, distribution, and reproduction in any medium, provided the original work is properly cited.

The field of graph theory is broadly growing and playing a remarkable role in cheminformatics, mainly in chemistry and mathematics in developing different chemical structures and their physicochemical properties. Mathematical chemistry provides a platform to study these physicochemical properties with the help of topological indices (TIs). A topological index (TI) is a function that connects a numeric number to each molecular graph. Zagreb indices (ZIs) are the most studied TIs. In this paper, we establish general expressions to calculate the connection-based multiplicative ZIs, namely, first multiplicative ZIs, second multiplicative ZIs, third multiplicative ZIs, and fourth multiplicative ZIs, of two renowned dendrimer nanostars. The defined expressions just depend on the step of growth of these dendrimers. Moreover, we have compared our calculated for both type of dendrimers with each other.

\section{Introduction}

TIs are the numerical numbers which are linked with different chemical structures of molecular graphs and predict the structural, toxicological, biological, and physicochemical properties of the existing chemical compounds. A graph in which the vertices represent atoms while the edges correspond to the covalent bonds between atoms is known as a molecular graph. TIs are extensively used in the study of quantitative structure-activity relationships and quantitative structure-property relationships [38]. Many researchers have worked on TIs $[1,18,25]$. TIs are classified into three distinct TIs, namely, degree-based TI, distance-based TI, and polynomial-based TI. A distance-based TI is a TI which is based on the distance between the vertices. In 1947, Wiener [39] developed the innovative conception of degree-based TI. Furthermore, Dankelmann et al. calculated the sharp upper bounds of graphs by utilizing these distance-based TIs in a very comprehensive way. Moreover, for the diameter $\delta \geq 2$, Mazorodze et al. [33] computed the sharp upper bounds of graphs by using the Gutman index which is also a distance-based TI. Furthermore, Fang et al. [15] discussed the topological properties of Sierpinski network along with its applications.

A degree-based TI is concerned with the degree of a vertex. Degree-based TI is further categorized into two subclasses named as degree and connection-based TIs. Gutman and Trinajstić [22] put forward the innovative idea of the well-known TI named as first Zagreb index (FZI). They used FZI to calculate the $\pi$-electron energy of the alternant hydrocarbon. Furthermore, second ZI was proposed by Gutman et al. [21] in 1975. The innovative idea of third ZI was proposed by Furtula and Gutman [17]. These first and second ZIs have been studied widely in distinct areas (see $[2,5,6])$. Chu et al. [10] calculated the sharp bounds of ZIs on connected graphs. Gharibi et al. [19] also worked on ZIs and investigated Zagreb polynomials of nanocone and nanotubes. Nikolic et al. [34] initiated modified ZIs in 2003. Hao [23] compared the ZIs and modified ZIs and discussed important results related to these indices in 2011. Furthermore, Dhanalakshmi et al. [12] introduced some modified and multiplicative ZIs (MZIs) on 
graph operators. Das et al. [11] in 2013 used MZIs to compute the upper bounds for some particular graphs. Fang et al. [14] computed ZIs of the hierarchical hypercube networks. For more details, we refer the readers to $[9,26,36]$.

Recently, the idea of connection number $(\mathrm{CN})$ has been instilled into the minds of researchers. $\mathrm{CN}$ is a number of those of vertices which are at distance 2 from a certain vertex. Ali and Trinajstić [4] investigated the modification of first ZI. In 2019, Tang et al. [37] developed some Zagreb connection indices (ZCIs). Furthermore, Ali et al. [3] computed modified ZCIs of T-sum graphs. Recently, In 2020, Liu et al. [32] gave Zagreb connection numbers of molecular graphs based on operations. Cao et al. [8] made use of ZCIs to calculate both exact and upper bounds of some product related graphs. Furthermore, Javaid et al. [28] initiated novel connection-based ZIs of different wheel related graphs. Haoer et al. [24] investigated the multiplicative leap ZIs.

A dendrimer is an artificially synthesized molecular structure made up of monomers (branched units). Dendrimer nanostars are highly branched nanostructures and are considered as the basic element in nanotechnology. The major three architectural parts of dendrimer nanostars are end groups, branches, and cores. Nowadays, dendrimer nanostars are rapidly gaining considerable attention from researchers due to their special chemical and physical characteristics and a broad range of applicability in distinct fields of bioscience, including drug delivery, immunology, and the advancement of antimicrobials, antivirals, and vaccines [29, 31]. Siddiqui et al. [35] introduced Zagreb polynomial of some nanostars in 2016. Furthermore, Bokhary et al. [7] discussed some molecular topological properties of dendrimers. In 2019, Fatima et al. [16] proposed ZCIs of two dendrimer nanostars in a very logical way. For more details about dendrimers, the readers are referred to $[13,30]$.

In this paper, we rewrite some already introduced connection-based MZIs. Further, we establish the general expressions to calculate the MZCIs of two well-known dendrimer nanostars in a very logical and comprehensive way. The proposed expressions only depend upon the step of growth of these dendrimers.

This paper is organized as follows. Section 1 presents some important definitions which are obligatory to understand the concept of our paper. In Section 2, we establish the general expression to find the connection-based MZIs of first type of dendrimer nanostar. Section 3 holds the general expression to calculate connection-based MZIs of the second type of dendrimer nanostar. Section 4 draws the conclusions.

\section{Preliminaries}

In this section, we define some basic definitions which are useful for the further evaluations.

Definition 1 (see [22]). Let $\zeta=(\mathbb{M}(\zeta), \mathbb{N}(\zeta))$ be a graph, where $\mathbb{M}(\zeta)$ and $\mathbb{N}(\zeta)$ represent the set of vertices and edges, respectively. Then, the first Zagreb index (FZI) can be given as

$$
\widehat{\mathbb{Z}}_{1}(\zeta)=\sum_{x \in \mathbb{M}(\zeta)}\left(\tilde{d}_{\zeta}(x)\right)^{2}
$$

This equation can be rewritten as

$$
\widehat{\mathbb{Z}}_{1}(\zeta)=\sum_{x y \in \mathbb{N}(\zeta)}\left(\tilde{d}_{\zeta}(x)+\tilde{d}_{\zeta}(y)\right),
$$

where $\widetilde{d}_{\zeta}(x)$ and $\widetilde{d}_{\zeta}(y)$ denote the degree of the vertices $x$ and $y$, respectively.

Definition 2 (see [21]). For graph $\zeta$, the second Zagreb index (SZI) can be given as

$$
\widehat{\mathbb{Z}}_{2}(\zeta)=\sum_{x y \in \mathbb{N}(\zeta)}\left(\tilde{d}_{\zeta}(x) \times \tilde{d}_{\zeta}(y)\right),
$$

where $\widetilde{d}_{\zeta}(x)$ and $\tilde{d}_{\zeta}(y)$ denote the degree of the vertices $x$ and $y$, respectively.

Definition 3 (see [4]). For a graph $\zeta$, the first Zagreb connection index (FZCI) and second Zagreb connection index (SZCI) can be given as

(1) $\hat{\mathbb{Z}} \mathfrak{C}_{1}(\zeta)=\sum_{x \in \mathbb{M}(\zeta)}\left(\tilde{\eta}_{\zeta}(x)\right)^{2}$

(2) $\hat{\mathbb{Z}} \mathfrak{S}_{2}(\zeta)=\sum_{x y \in \mathbb{N}(\zeta)}\left(\tilde{\eta}_{\zeta}(x) \times \tilde{\eta}_{\zeta}(y)\right)$, where $\tilde{\eta}_{\zeta}(x)$ and $\tilde{\eta}_{\zeta}(y)$ denote the connection number of the vertices $x$ and $y$, respectively.

Definition 4 (see [4]). For a graph $\zeta$, the modified first Zagreb connection index can be given as

$$
\hat{\mathbb{Z}}_{1}^{*}(\zeta)=\sum_{x \in \mathbb{M}(\zeta)}\left(\tilde{\eta}_{\zeta}(x)+\eta_{\zeta}(x)\right) .
$$

Definition 5 (see [20]). For a graph $\zeta$, the first multiplicative Zagreb index (FMZI) and second Zagreb index (SMZI) can be given as
(1) $\hat{\mathbb{Z}}_{1}^{\mathbb{M}}(\zeta)=\prod_{x y \in \mathbb{N}(\zeta)}\left(\widetilde{d}_{\zeta}(x)+\widetilde{d}_{\zeta}(y)\right)$
(2) $\widehat{\mathbb{Z}}_{2}^{\mathbb{M}}(\zeta)=\prod_{x y \in \mathbb{N}(\zeta)}\left(\widetilde{d}_{\zeta}(x) \times \widetilde{d}_{\zeta}(y)\right)$

Definition 6 (see [27]). For a graph $\zeta$, connection-based MZIs can be defined as

(1) $\widehat{\mathbb{Z}}_{1}^{\mathbb{M}} \mathfrak{E}(\zeta)=\prod_{x \in \mathbb{M}(\zeta)}\left(\tilde{\eta}_{\zeta}(x)\right)^{2}$ (first MZCI)

(2) $\widehat{\mathbb{Z}}_{2}^{\mathbb{M}} \mathfrak{S}(\zeta)=\prod_{x y \in \mathbb{N}(\zeta)}\left(\tilde{\eta}_{\zeta}(x) \times \tilde{\eta}_{\zeta}(y)\right)$ (second MZCI)

(3) $\hat{\mathbb{Z}}_{3}^{\mathbb{M}} \mathfrak{E}(\zeta)=\prod_{x \in \mathbb{M}(\zeta)}\left(\tilde{d}_{\zeta}(x) \times \tilde{\eta}_{\zeta}(x)\right)$ (third MZCI)

(4) $\widehat{\mathbb{Z}}_{4}^{\mathbb{M}} \mathfrak{S}(\zeta)=\prod_{x y \in \mathbb{N}(\zeta)}\left(\tilde{\eta}_{\zeta}(x)+\tilde{\eta}_{\zeta}(y)\right)$ (fourth MZCI)

Now, before moving towards our main results of this article, first we rewrite the connection-based MZIs given in Definition 6.

Definition 7. For a graph $\zeta$, the first MZCI can be rewritten as 


$$
\widehat{\mathbb{Z}}_{1}^{\mathbb{M}} \mathfrak{C}(\zeta)=\prod_{0 \leq \alpha \leq \widehat{n}-2}\left[\alpha^{2}\right]^{\mathscr{C}_{\alpha}(\zeta)}
$$

where $\mathscr{C}_{\alpha}(\zeta)$ is the total number of vertices in $\zeta$ with connection number $\alpha$.

The second MZCI can be rewritten as

$$
\widehat{\mathbb{Z}}_{2}^{\mathbb{M}} \mathfrak{C}(\zeta)=\prod_{0 \leq \alpha \leq \beta \leq \hat{n}-2}[\alpha \times \beta]^{\mathscr{C}_{(\alpha, \beta)}(\zeta)},
$$

where $\mathscr{C}_{(\alpha, \beta)}(\zeta)$ is the total number of edges in $\zeta$ with connection numbers $(\alpha, \beta)$.

Similarly, the third MZCI can be given as

$$
\widehat{\mathbb{Z}}_{3}^{\mathbb{M}} \mathfrak{E}(\zeta)=\prod_{0 \leq \gamma \leq \alpha \leq \widehat{n}-2}[\gamma \times \alpha]^{\mathscr{C}_{(\gamma, \alpha)}^{\prime}(\zeta)},
$$

where $\mathscr{C}_{(\gamma, \alpha)}^{\prime}(\zeta)$ is the total number of vertices with degree $\gamma$ and $\mathrm{CN} \alpha$.

The fourth MZCI can be written as

$$
\widehat{\mathbb{Z}}_{4}^{\mathbb{M}} \mathfrak{S}(\zeta)=\prod_{0 \leq \alpha \leq \beta \leq \widehat{n}-2}[\alpha+\beta]^{\mathscr{C}_{(\alpha, \beta)}(\zeta)} .
$$

\section{MZCIs of First Type of Dendrimer Nanostar}

In this section, we establish the general expressions to calculate the MZCIs of first type of dendrimer nanostar. First, we provide the construction of the dendrimer nanostar of the first type, i.e., $\mathbb{D}_{1}[\widehat{m}]$, by labeling the vertices with degrees and $\mathrm{CNs}$. The skeletal formulas of $\mathbb{D}_{1}[\widehat{m}]$ along with connection number for $\widehat{m}=1,2,3$ are shown in Figures 1-3.

The skeletal formulas of dendrimer nanostar $\mathbb{D}_{1}[\widehat{m}]$ along with degrees are shown in Figures 4-6.

Before presenting the main results our paper, we first classify the hexagons of $\mathbb{D}_{1}[\widehat{m}]$ with the help the degrees of the vertices into terminal hexagon, initial hexagon, and $\lambda$-hexagon.

(i) Terminal Hexagon. A hexagon in which the degree of exactly five vertices is two is said to be terminal hexagon.

(ii) Initial Hexagon. A hexagon which is in the center of $\mathbb{D}_{1}[\widehat{m}]$ is said to be initial hexagon.

(iii) $\lambda$-Hexagon. A hexagon which is neither initial nor terminal is said to be $\lambda$-hexagon.

All the remaining vertices which do not lie in any of the above mentioned hexagons are said to be $\mu$-type vertices. By $(r, s)$-type edges, we mean the edge joining the vertices with CNs $r$ and $s$.

Theorem 1. Let $\zeta=\mathbb{D}_{1}[\hat{m}]$ be a molecular graph for $\widehat{m}=1,2,3$. Then, first MZCI and third MZCI of $\zeta$ are given below:
(1) $\widehat{\mathbb{Z}}_{1}^{\mathbb{M}}(\zeta)=(64)^{\kappa}(489776025600)^{\kappa-2}$
(2) $\widehat{\mathbb{Z}}_{3}^{\mathbb{M}}(\zeta)=(64)^{\kappa}(7255941120)^{(\kappa-2)}$

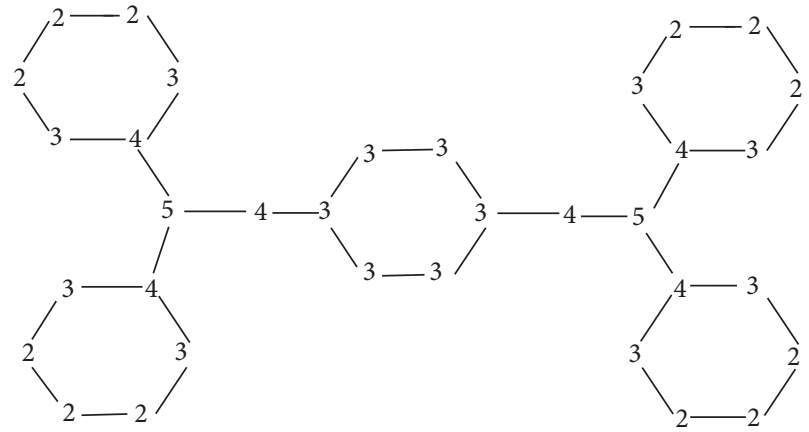

Figure 1: $\mathbb{D}_{1}[1]$ along with the connection of each vertex.

where $\kappa=2^{\widehat{m}+1}$.

Proof.

(1) First, we find the total number of terminal hexagons, initial hexagons, and $\lambda$-hexagons. By simple observation, we have

$$
\begin{aligned}
\text { total number of terminal hexagons } & =2^{\widehat{m}+1}, \\
\text { total number of initial hexagons } & =1 .
\end{aligned}
$$

The total number of $\lambda$-hexagons in $\zeta$ for $\widehat{m}=1,2,3, \cdots$ is $0,4,12,28, \ldots$, respectively. The $n^{\text {th }}$ term of the sequence is $2^{\hat{m}+1}-4$. Thus,

$$
\text { total number of } \lambda \text { - hexagons }=2^{\widehat{m}+1}-4 \text {. }
$$

The total number of $\mu$ - type vertices in $\zeta$ for $\widehat{m}=$ $1,2,3, \cdots$ is $4,12,28, \ldots$, respectively. The $n^{\text {th }}$ term of the sequence is $2 \times 2^{\widehat{m}+1}-4$. Thus,

total number of $\mu-$ vertices $=2 \times 2^{\widehat{m}+1}-4$.

Now, we find the number of vertices having CN 2 in $\zeta$, i.e., $\mathscr{C}_{2}(\zeta)$. One can observe easily that all those vertices which have CN 2 are present only in terminal hexagons and no vertices exist having $\mathrm{CN} 2$ in central and $\lambda$-hexagons. Every terminal hexagon has exactly 3 vertices with $\mathrm{CN}_{2}$ and the total number of terminal hexagons in $\zeta$ is $2^{m+1}$. Thus, $\mathscr{C}_{2}(\zeta)$ must be equal to 3 times the number of terminal hexagons in $\zeta$. Mathematically, we have

$$
\mathscr{C}_{2}(\zeta)=3 \times 2^{\widehat{m}+1}
$$

Now, we find the number of vertices having CN 3 in $\zeta$, i.e., $\mathscr{C}_{3}(\zeta)$. In Table 1 , we have calculated the total number of vertices with $\mathrm{CN} 3$ in $\zeta$.

The total number of vertices with $\mathrm{CN} 3$ in $\zeta$ is the sum of the number of vertices with $\mathrm{CN} 3$ in terminal hexagon, initial hexagon, and $\lambda$-hexagon of $\zeta$. So, 


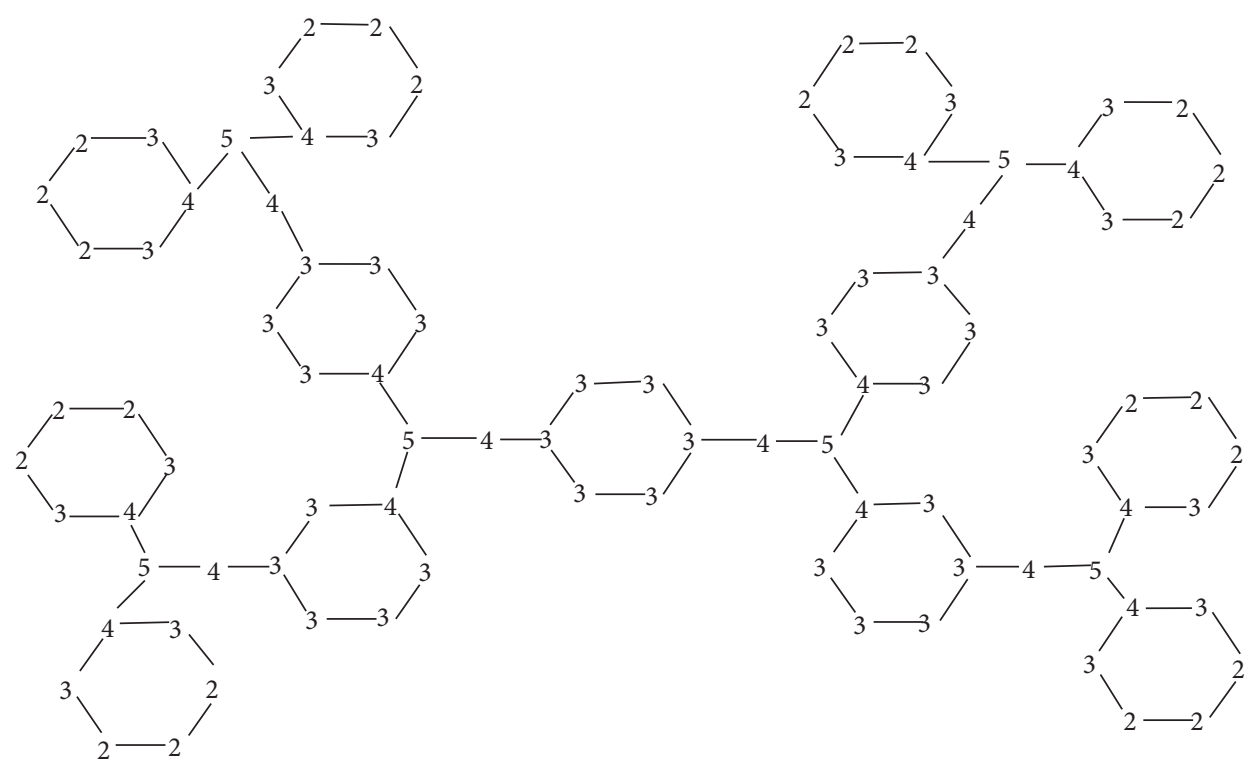

FIGURE 2: $\mathbb{D}_{1}[2]$ along with the connection of each vertex.

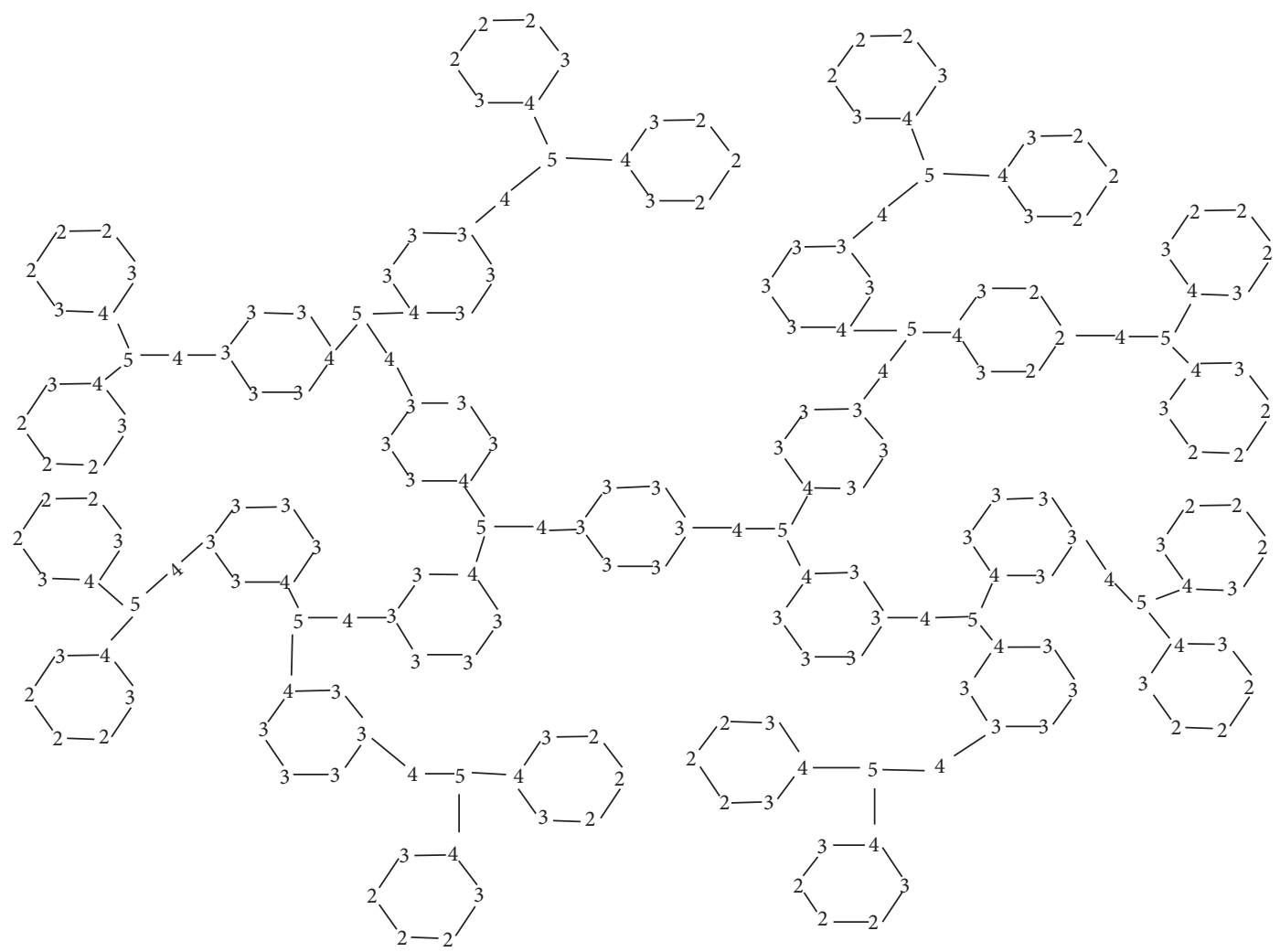

FIGURE 3: $\mathbb{D}_{1}[1]$ along with the degree of each vertex.

$$
\begin{aligned}
\mathscr{C}_{3}(\zeta) & =2 \times 2^{\widehat{m}+1}+5\left(2^{\hat{m}+1}-4\right)+6 \\
& =2 \times 2^{\widehat{m}+1}+5\left(2^{\hat{m}+1}\right)-20+6 \\
& =7 \times 2^{\widehat{m}+1}-14 .
\end{aligned}
$$

Next, we calculate the total number of vertices with $\mathrm{CN} 4$ in $\zeta$, i.e., $\mathscr{C}_{4}(\zeta)$. Table 2 shows the total number of vertices with $\mathrm{CN} 4$ in $\zeta$.

On can easily observe that half of the $\mu$-type vertices have $\mathrm{CN} 4$ while the other half has $\mathrm{CN} 5$. We know that the total number of $\mu$-type vertices is 


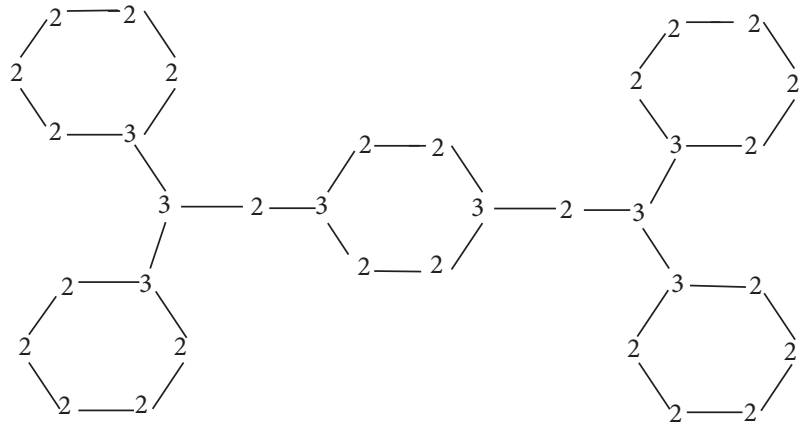

Figure $4: \mathbb{D}_{1}[2]$ along with the degree of each vertex.

$2 \times 2^{\widehat{m}+1}-4$. Thus, the number of vertices having $\mathrm{CN}$ 4 must be $2^{\hat{m+1}}-2$. The total number of vertices with $\mathrm{CN} 4 \mathrm{in} \zeta$ is the sum of the number of vertices with $\mathrm{CN} 4$ in terminal hexagon, initial hexagon, $\lambda$-hexagon, and $\mu$-type vertices of $\zeta$. So,

$$
\begin{aligned}
\mathscr{C}_{4}(\zeta) & =2^{\widehat{m}+1}+0+\left(2^{\widehat{m}+1}-4\right)+\left(2^{\widehat{m}+1}-2\right) \\
& =3 \times 2^{\widehat{m}+1}-6 .
\end{aligned}
$$

Finally, we calculate $\mathscr{C}_{5}(\zeta)$. So,

$$
\mathscr{C}_{5}(\zeta)=2^{\hat{m}+1}-2 .
$$

By using equation (5), we have

$$
\begin{aligned}
\widehat{\mathbb{Z}}_{1}^{\mathbb{M}} \mathfrak{C}(\zeta) & =\prod_{0 \leq \alpha \leq \widehat{n}-2}\left[\alpha^{2}\right]^{\mathscr{C}_{\alpha}(\zeta)} \\
& =\left[2^{2}\right]^{\mathscr{C}_{2}(\zeta)} \times\left[3^{2}\right]^{\mathscr{C}_{3}(\zeta)} \times\left[4^{2}\right]^{\mathscr{C}_{4}(\zeta)} \times\left[5^{2}\right]^{\mathscr{C}_{5}(\zeta)} \\
& =\left[2^{2}\right]^{3 \times 2^{m+1}} \times\left[3^{2}\right]^{7 \times 2^{(m+1)}-14} \times\left[4^{2}\right]^{3 \times 2^{(m+1)}-6} \times\left[5^{2}\right]^{2^{m+1}-2} \\
& =(64)^{\kappa} \times(489776025600)^{\kappa-2},
\end{aligned}
$$

where $\kappa=2^{\widehat{m}+1}$.

(2) Firstly, we calculate number of vertices with degree 2 and $\mathrm{CN} 2$, i.e., $\mathscr{C}_{(2,2)}^{\prime}(\zeta)$. The number of vertices with degree 2 and $\mathrm{CN} 2$ in terminal hexagon, initial hexagon, and $\lambda$-type hexagon is given in Table 3 .
Thus, we have

$$
\mathscr{C}_{(2,2)}^{\prime}(\zeta)=3 \times 2^{\widehat{m}+1}
$$

Now, we calculate the number of vertices with degree 2 and $\mathrm{CN} 3$ in $\zeta$. The number of vertices with degree 2 and $\mathrm{CN} 3$ in terminal hexagon, initial hexagon, and $\lambda$-type hexagon is given in Table 4 .

Thus, $\mathscr{C}_{(2,3)}^{\prime}(\zeta)$ will be

$$
\begin{aligned}
\mathscr{C}_{(2,3)}^{\prime}(\zeta) & =2 \times 2^{\widehat{m}+1}+4\left(2^{\widehat{m}+1}-4\right)+4 \\
& =6 \times 2^{\widehat{m}+1}-12 .
\end{aligned}
$$

We notice that the number of vertices with degree 2 and $\mathrm{CN} 4$ is present only in half of the $\mu$-type vertices of $\zeta$. Thus, $\mathscr{C}_{(2,4)}{ }^{\prime}(\zeta)$ will be

$$
\mathscr{C}_{(2,4)}^{\prime}(\zeta)=2^{\widehat{m}+1}
$$

Now, we calculate the number of vertices with degree 3 and $\mathrm{CN} 3$ in $\zeta$. The number of vertices with degree 3 and $\mathrm{CN}$ 3 in terminal hexagon, initial hexagon, and $\lambda$-type hexagon is given in Table 5.

Thus, $\mathscr{C}_{(3,3)}^{\prime}(\zeta)$ will be

$$
\begin{aligned}
\mathscr{C}_{(3,3)}^{\prime}(\zeta) & =2+2^{\widehat{m}+1}-4 \\
& =2^{\widehat{m}+1}-2 .
\end{aligned}
$$

Similarly,

$$
\mathscr{C}_{(3,5)}^{\prime}(\zeta)=2^{\widehat{m}+1}-2 .
$$

The number of vertices with degree 3 and $\mathrm{CN} 4$ in terminal hexagon, initial hexagon, and $\lambda$-type hexagon is given in Table 6 .

Thus, $\mathscr{C}_{(3,4)}^{\prime}(\zeta)$ will be

$$
\begin{aligned}
\mathscr{C}_{(3,4)}^{\prime}(\zeta) & =2^{\widehat{m}+1}+2^{\widehat{m}+1}-4+4 \\
& =2 \times 2^{\widehat{m}+1}-4 .
\end{aligned}
$$

By substituting all the values of $\mathscr{C}_{(\alpha, \beta)}^{\prime}(\zeta)$ in equation (7), we have

$$
\begin{aligned}
\widehat{\mathbb{Z}}_{3}^{\mathbb{M}} \mathfrak{S}(\zeta) & =\prod_{0 \leq \gamma \leq \alpha \leq \widehat{m}-2}[\alpha \times \beta]^{\mathscr{C}_{(\alpha, \beta)}^{\prime}(\zeta)} \\
& =[4]^{\mathscr{C}_{(2,2)}^{\prime}(\zeta)} \times[6]^{\mathscr{C}_{(2,3)}^{\prime}(\zeta)} \times[8]^{\mathscr{C}_{(2,4)}^{\prime}(\zeta)} \times[9]^{\mathscr{C}_{(3,3)}^{\prime}(\zeta)} \times[12]^{\mathscr{C}_{(3,4)}^{\prime}(\zeta)} \times[20]^{\mathscr{C}_{(3,5)}^{\prime}(\zeta)} \\
& =[4]^{3 \times 2^{2^{m+1}}} \times[6]^{6 \times 2^{m+1}-12} \times[8]^{2^{m+1}-2} \times[9]^{2^{2^{m}+1}-2} \times[12]^{2 \times 2^{m+1}-4} \times[15]^{2^{m+1}-2} \\
& =[4]^{3 \kappa} \times[6]^{6(\kappa-2)} \times[8]^{\kappa-2} \times[9]^{\kappa-2} \times[12]^{2 \times(\kappa-2)} \times[15]^{\kappa-2} \\
& =(64)^{\kappa}(7255941120)^{(\kappa-2)},
\end{aligned}
$$




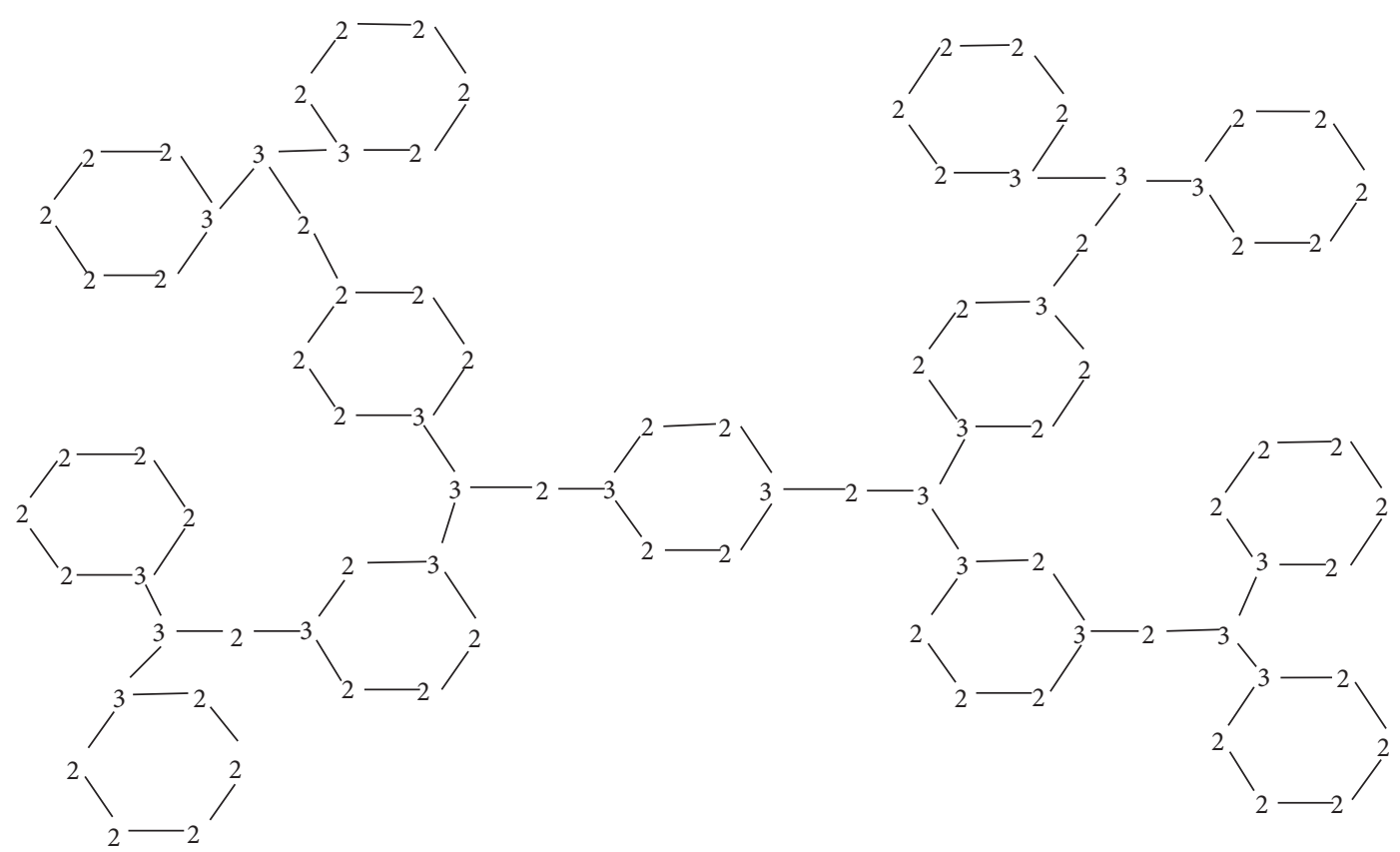

FIGURE 5: $\mathbb{D}_{1}[2]$ along with the degree of each vertex.

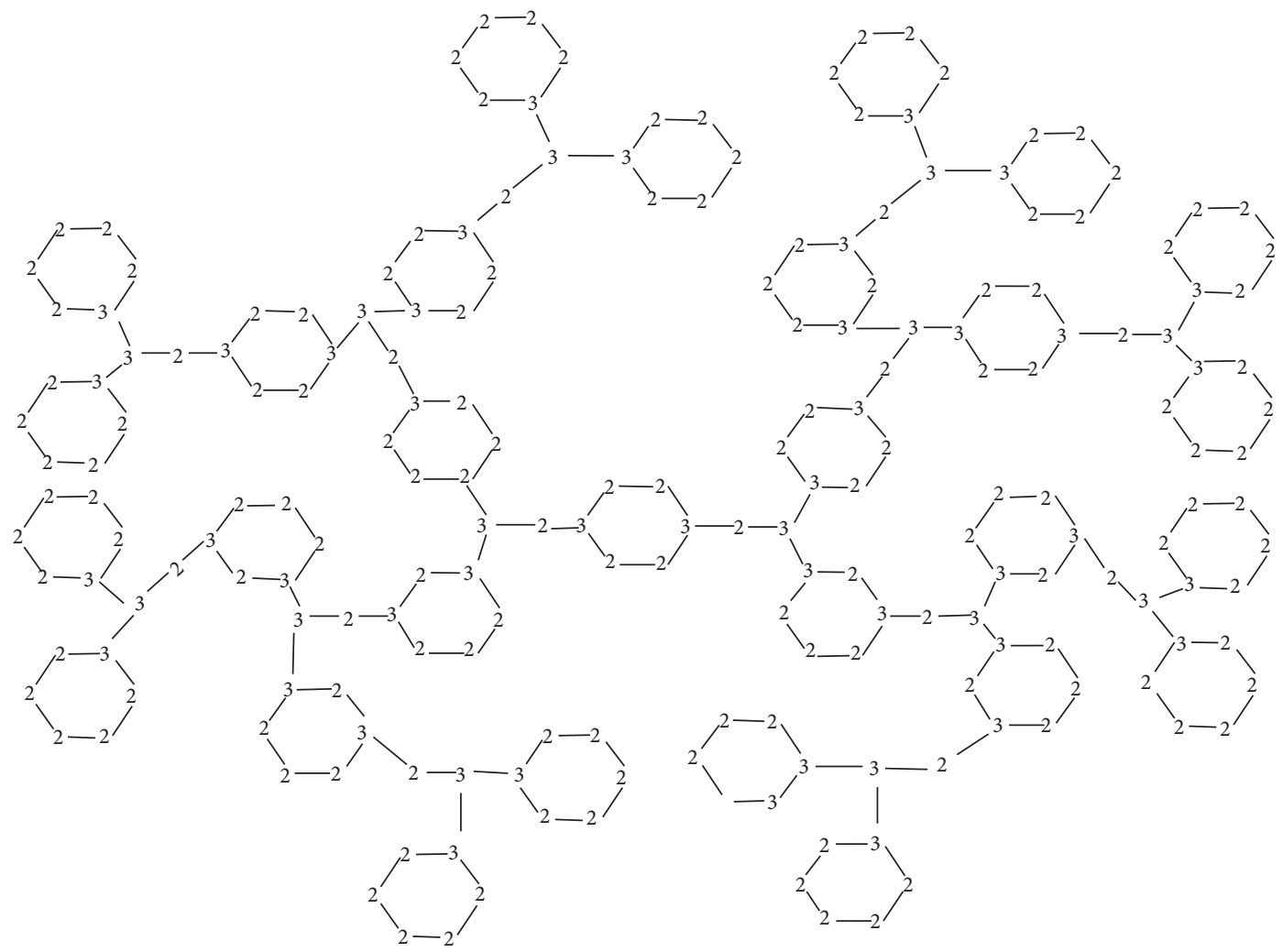

Figure 6: $\mathbb{D}_{1}[3]$ along with the degree of each vertex. 
TABLE 1: Total number of vertices with CN 3 in $\zeta$.

\begin{tabular}{lccc}
\hline $\begin{array}{l}\text { Types of } \\
\text { hexagons }\end{array}$ & $\begin{array}{c}\text { Number of vertices with CN 3 in } \\
\text { hexagon }(x)\end{array}$ & $\begin{array}{c}\text { Total number of hexagons } \\
(y)\end{array}$ & $\begin{array}{c}\text { Total number of vertices with CN } 3 \text { in hexagons } \\
(x \times y)\end{array}$ \\
\hline Terminal & 2 & $2^{\widehat{m}+1}$ & $2 \times 2^{\widehat{m}+1}$ \\
Initial & 6 & 1 & 6 \\
$\lambda$ & 5 & $2^{\widehat{m}+1}-4$ & $5\left(2^{\widehat{m}+1}-4\right)$ \\
$\mu$-type & 0 & 0 & 0 \\
\hline
\end{tabular}

TABLE 2: Total number of vertices with CN 4 in $\zeta$.

\begin{tabular}{lccc}
\hline $\begin{array}{l}\text { Types of } \\
\text { hexagons }\end{array}$ & $\begin{array}{c}\text { Number of vertices with CN } 4 \text { in } \\
\text { hexagon }(x)\end{array}$ & $\begin{array}{c}\text { Total number of hexagons } \\
(y)\end{array}$ & $\begin{array}{c}\text { Total number of vertices with CN } 4 \text { in hexagons } \\
(x \times y)\end{array}$ \\
\hline Terminal & 1 & $2^{\widehat{m}+1}$ & $2^{\hat{m}+1}$ \\
Initial & 0 & 1 & 0 \\
$\lambda$ & 1 & $2^{\widehat{m}+1}-4$ & $2^{\widehat{m}+1}-4$ \\
\hline
\end{tabular}

TABle 3: Total number of vertices with degree 2 and CN 2 in $\zeta$.

\begin{tabular}{lccc}
\hline $\begin{array}{l}\text { Types of } \\
\text { hexagons }\end{array}$ & $\begin{array}{c}\text { Number of vertices with degree 2 and CN 2 } \\
\text { in hexagon }(x)\end{array}$ & $\begin{array}{c}\text { Total number of } \\
\text { hexagons }(y)\end{array}$ & $\begin{array}{c}\text { Total number of vertices with degree } 2 \text { and CN } 2 \text { in } \\
\text { hexagons }(x \times y)\end{array}$ \\
\hline Terminal & 3 & $2^{\hat{m}+1}$ & $3 \times 2^{\hat{m}+1}$ \\
Initial & 0 & 1 & 0 \\
$\lambda$ & 0 & $2^{\hat{m}+1}-4$ & 0 \\
\hline
\end{tabular}

TABLE 4: Total number of vertices with degree 2 and CN 3 in $\zeta$.

\begin{tabular}{lccc}
\hline $\begin{array}{l}\text { Types of } \\
\text { hexagons }\end{array}$ & $\begin{array}{c}\text { Number of vertices with degree 2 and CN 3 } \\
\text { in hexagon }(x)\end{array}$ & $\begin{array}{c}\text { Total number of } \\
\text { hexagons }(y)\end{array}$ & $\begin{array}{c}\text { Total number of vertices with degree } 2 \text { and CN } 3 \text { in } \\
\text { hexagons }(x \times y)\end{array}$ \\
\hline Terminal & 2 & $2^{m+1}$ & $2 \times 2^{\widehat{m}+1}$ \\
Initial & 4 & 1 & 4 \\
$\lambda$ & 4 & $2^{\widehat{m}+1}-4$ & $4\left(2^{\widehat{m}+1}-4\right)$ \\
\hline
\end{tabular}

where $\kappa=2^{\widehat{m}+1}$.

Theorem 2. Let $\zeta=\mathbb{D}_{1}[\hat{m}]$ be a molecular graph for $\widehat{m}=1,2,3$. Then, second MZCI and fourth MZCI of $\zeta$ are given below

(1) $\widehat{Z}_{2}^{\mathbb{M}} \mathfrak{C}(\zeta)=(576)^{\kappa}(1990656000)^{(\kappa-2)}(81)^{(2 \kappa-5)}$,

(2) $\widehat{Z}_{4}^{\mathbb{M}} \mathfrak{S}(\zeta)=(400)^{\kappa}(12252303)^{(\kappa-2)}(36)^{2 \kappa-5}$, where $\kappa=2^{\widehat{m}+1}$.

Proof.

(1) First, we calculate $\mathscr{C}_{2,2}(\zeta)$, i.e., $(2,2)$-type edges in $\zeta$. One can easily observe that graph $\zeta$ has $(2,2)$-type edges only in terminal hexagon. There are exactly two (2,2)-type and (2,3)-type edges in every terminal hexagon of $\zeta$. Hence, $\mathscr{C}_{2,2}(\zeta)$ must be equal to

$$
\begin{aligned}
& \mathscr{C}_{(2,2)}(\zeta)=2 \times 2^{\widehat{m}+1}, \\
& \mathscr{C}_{(2,3)}(\zeta)=2 \times 2^{\widehat{m}+1} .
\end{aligned}
$$

Next, the total number of $(3,3)$-type edges is displayed in Table 7.
The total number of $(3,3)$-type edges in $\zeta$ is the sum of the $(3,3)$-type edges in terminal hexagon, initial hexagon, $\lambda$-hexagon of $\zeta$. So,

$$
\mathscr{C}_{(3,3)}(\zeta)=4 \times 2^{\widehat{m}+1}-10 .
$$

Now, we calculate $\mathscr{C}_{(3,4)}(\zeta)$. The total number of $(3,4)$-type edges in every terminal hexagon, initial hexagon, and $\lambda$-hexagons of $\zeta$ is displayed in Table 8 .

The total number of $(3,4)$-type edges which do not exist in any of the hexagon of $\zeta$ is equal to the number of $\mu$-type vertices with CN 4 . Thus, $\mathscr{C}_{(3,4)}(\zeta)$ will be

$$
\begin{aligned}
\mathscr{C}_{(3,4)}(\zeta) & =2 \times 2^{\widehat{m}+1}+2\left(2^{\widehat{m}+1}-4\right)+\left(2^{\widehat{m}+1}-2\right) \\
& =5 \times 2^{\widehat{m}+1}-10 .
\end{aligned}
$$

Finally, we calculate $\mathscr{C}_{(4,5)}(\zeta)$. We observe that $(4,5)$-type edges in $\zeta$ are equal to 3 times the number of $\mu$-type vertices of $\zeta$ with CN 5 . Thus, we have 
TABLE 5: Total number of vertices with degree 3 and $\mathrm{CN} 3$ in $\zeta$.

\begin{tabular}{lccc}
\hline $\begin{array}{l}\text { Types of } \\
\text { hexagons }\end{array}$ & $\begin{array}{c}\text { Number of vertices with degree 3 and CN 3 } \\
\text { in hexagon }(x)\end{array}$ & $\begin{array}{c}\text { Total number of } \\
\text { hexagons }(y)\end{array}$ & $\begin{array}{c}\text { Total number of vertices with degree 3 and CN 3 in } \\
\text { hexagons }(x \times y)\end{array}$ \\
\hline Terminal & 0 & $2^{\widehat{m}+1}$ & 0 \\
Initial & 2 & $2^{\widehat{m}+1}-4$ & 2 \\
$\lambda$ & 1 & $2^{\hat{m}+1}-4$ & 4 \\
\hline
\end{tabular}

TABle 6: Total number of vertices with degree 3 and $\mathrm{CN} 4$ in $\zeta$.

\begin{tabular}{lccc}
\hline $\begin{array}{l}\text { Types of } \\
\text { hexagons }\end{array}$ & $\begin{array}{c}\text { Number of vertices with degree 3 and CN 4 } \\
\text { in hexagon }(x)\end{array}$ & $\begin{array}{c}\text { Total number of } \\
\text { hexagons }(y)\end{array}$ & $\begin{array}{c}\text { Total number of vertices with degree } 3 \text { and CN } 4 \text { in } \\
\text { hexagons }(x \times y)\end{array}$ \\
\hline Terminal & 1 & $2^{\widehat{m}+1}$ & $2^{\widehat{m}+1}$ \\
Initial & 0 & 1 & $\left(2^{\widehat{m}+1}-4\right)$ \\
$\lambda$ & 1 & $2^{\widehat{m}+1}-4$ & 0 \\
\hline
\end{tabular}

$$
\mathscr{C}_{(4,5)}(\zeta)=3\left(2^{\hat{m}+1}-2\right)
$$

By using equation (6), we have

$$
\begin{aligned}
\widehat{\mathbb{Z}}_{2}^{\mathbb{M}} \mathfrak{C}(\zeta) & =\prod_{0 \leq \alpha \leq \beta \leq \widehat{n}-2}[\alpha \times \beta]^{\mathscr{C}_{(\alpha, \beta)}(\zeta)} \\
& =[4]^{\mathscr{C}_{(2,2)}(\zeta)} \times[6]^{\mathscr{C}_{(2,3)}(\zeta)} \times[9]^{\mathscr{C}_{(3,3)}(\zeta)} \times[12]^{\mathscr{C}_{(3,4)}(\zeta)} \times[20]^{\mathscr{C}_{(4,5)}(\zeta)} \\
& =[4]^{2 \times 2^{\hat{m}+1}} \times[6]^{2 \times 2^{m+1}} \times[9]^{4 \times 2^{m+1}-10} \times[12]^{5 \times 2^{\hat{m}+1}-10} \times[20]^{3\left(2^{m+1}-2\right)} \\
& =[4]^{2 \kappa} \times[6]^{2 \kappa} \times[9]^{4 \kappa-10} \times[12]^{5 \kappa-10} \times[20]^{3 \kappa-6} \\
& =(576)^{\kappa}(1990656000)^{(\kappa-2)}(81)^{(2 \kappa-5)},
\end{aligned}
$$

where $\kappa=2^{\widehat{m}+1}$.

$$
\begin{aligned}
\widehat{\mathbb{Z}}_{4}^{\mathbb{M}} \mathfrak{C}(\zeta) & =\prod_{0 \leq \alpha \leq \beta \leq \widehat{m}-2}[\alpha+\beta]^{\mathscr{C}_{(\alpha, \beta)}(\zeta)} \\
& =[4]^{\mathscr{C}_{(2,2)}(\zeta)} \times[5]^{\mathscr{C}_{(2,3)}(\zeta)} \times[6]^{\mathscr{C}_{(3,3)}(\zeta)} \times[7]^{\mathscr{C}_{(3,4)}(\zeta)} \times[9]^{\mathscr{C}_{(4,5)}(\zeta)} \\
& =[4]^{2 \times 2^{\widehat{m}+1}} \times[5]^{2 \times 2^{\widehat{m}+1}} \times[6]^{4 \times 2^{\widehat{m+1}}-10} \times[7]^{5 \times 2^{\widehat{m}+1}-10} \times[9]^{3\left(2^{\widehat{m}+1}-2\right)} \\
& =(400)^{\kappa}(12252303)^{(\kappa-2)}(36)^{2 \kappa-5}
\end{aligned}
$$

\section{MZCIs of Second Type of Dendrimer Nanostar}

In this section, we calculate the multiplicative ZCIs of second type of dendrimer nanostars. First, we provide the construction of the dendrimer nanostar of the second type, i.e., $\mathbb{D}_{2}[\widehat{m}]$, by labeling the vertices with degrees and CNs. The skeletal formulas of $\mathbb{D}_{2}[\widehat{m}]$ along with connection number for $\widehat{m}=1,2,3$ are shown in Figures 7-9.

The skeletal formulas of dendrimer nanostar $\mathbb{D}_{2}[\widehat{m}]$ along with degrees of each vertex are shown in Figures 10-12.
Before stating our main results about the second type of dendrimers, we classify hexagons and pentagons of $\mathbb{D}_{2}[\mathrm{~m}]$, for $m=1,2,3$, with the help of degrees of the vertices, into the following:

(1) Terminal Pentagon. A pentagon which has three adjacent vertices of degree 1 .

(2) Non-Terminal Pentagon. A pentagon which is not terminal is said to be non-terminal pentagon.

(3) $\eta$-Hexagon. A hexagon which has exactly two vertices of degree 3. 
TABLE 7: The total number of (3,3)-type edges in hexagons of $\zeta$.

\begin{tabular}{lccc}
\hline $\begin{array}{l}\text { Types of } \\
\text { hexagons }\end{array}$ & $\begin{array}{c}\text { Number of (3,3)-type edges in hexagon } \\
(x)\end{array}$ & $\begin{array}{c}\text { Total number of hexagons } \\
(y)\end{array}$ & $\begin{array}{c}\text { Total number of }(3,3) \text {-type edges in hexagons } \\
(x \times y)\end{array}$ \\
\hline Terminal & 0 & $2^{\hat{m}+1}$ & 0 \\
Initial & 6 & 1 & $4\left(2^{\hat{m}+1}-4\right)$ \\
$\lambda$ & 4 & $2^{\hat{m}+1}-4$ & 6 \\
\hline
\end{tabular}

TABLE 8: The total number of $(3,4)$-type edges in hexagons of $\zeta$.

\begin{tabular}{lccc}
\hline $\begin{array}{l}\text { Types of } \\
\text { hexagons }\end{array}$ & $\begin{array}{c}\text { Number of (3,4)-type edges in hexagon } \\
(x)\end{array}$ & $\begin{array}{c}\text { Total number of hexagons } \\
(y)\end{array}$ & $\begin{array}{c}\text { Total number of }(3,4) \text {-type edges in hexagons } \\
(x \times y)\end{array}$ \\
\hline Terminal & 2 & $2^{\widehat{m}+1}$ & $2 \times 2^{\hat{m}+1}$ \\
Initial & 0 & 1 & $2\left(2^{\widehat{m}+1}-4\right)$ \\
$\lambda$ & 2 & $2^{\widehat{m}+1}-4$ & 0 \\
\hline
\end{tabular}

TABle 9: Types of pentagons and hexagons.

\begin{tabular}{lc}
\hline $\begin{array}{l}\text { Types of pentagons and } \\
\text { hexagons }\end{array}$ & Total number of pentagons in $\zeta$ \\
\hline Terminal pentagon & 1 \\
Non-terminal pentagon & $2^{\widehat{m}}-1$ \\
$\eta$-hexagon & $2^{\hat{m}}$ \\
$\phi$-hexagon & $2\left(2^{\widehat{m}-1}-1\right)$ \\
$\vartheta$-hexagon & $2\left(2^{\widehat{m}}-1\right)$ \\
$\psi$-hexagon & $2^{\widehat{m}}-1$ \\
\hline
\end{tabular}

(4) $\phi$-Hexagon. A hexagon which has exactly three vertices of degree 3.

(5) 9 -Hexagon. A hexagon which has exactly four vertices of degree 3.

(6) $\psi$-Hexagon. A hexagon which has exactly six vertices of degree 3.

All those vertices which do not lie in any type of pentagon or hexagon are said to be $\mu$-type vertices.

Theorem 3. Let $\zeta=\mathbb{N}_{2}[\widehat{m}]$ be a molecular graph of order $\widehat{n}$. Then, the first and third MZCIs of a molecular graph $\zeta$ are given as

(1) $\widehat{\mathbb{Z}}_{1}^{\mathbb{M}}(\zeta)=(4)^{4 \kappa-1}(16)^{6 \kappa-7}(16815125390625)^{\kappa-1}$ $(36)^{5 \kappa-6}$

(2) $\widehat{\mathbb{Z}}_{3}^{\mathbb{M}}(\zeta)=(16)^{\kappa}(2)^{2 \kappa-1}(5015306502144)^{\kappa-1}(15)^{9 \kappa-10}$ $(18)^{5(2 \kappa-3)}$,

where $\kappa=2^{\hat{m}}$.

Proof. First, we find the total number of hexagons and pentagons. The total number of pentagons and hexagons of a graph $\zeta$ is depicted in Table 9.

The vertices which do not exist in any of the abovedefined hexagon is said to be $\mu$-type vertices. The total number of $\mu$-type vertices is $2^{\widehat{m}+1}-1$.
(1) After simple calculation, the total number of vertices with CNs 2, 3, 4, 5, and 6, from Figure 9, is

$$
\begin{aligned}
& \mathscr{C}_{2}(\zeta)=4 \times 2^{\widehat{m}}-1, \\
& \mathscr{C}_{3}(\zeta)=8\left(2^{\widehat{m}}-1\right), \\
& \mathscr{C}_{4}(\zeta)=6 \times 2^{\widehat{m}}-7, \\
& \mathscr{C}_{5}(\zeta)=4\left(2^{\widehat{m}}-1\right), \\
& \mathscr{C}_{6}(\zeta)=5 \times 2^{\widehat{m}}-6 .
\end{aligned}
$$

By putting the above values in equation (5), we have

$$
\widehat{\mathbb{Z}}_{1}^{\mathbb{M}}(\zeta)=(4)^{4 \kappa-1}(16)^{6 \kappa-7}(16815125390625)^{\kappa-1}(36)^{5 \kappa-6} .
$$

(2) Now, we find $\mathscr{C}_{(\gamma, \alpha)}{ }^{\prime}(\zeta)$. From Figures 9 and 12, we have

$$
\begin{aligned}
& \mathscr{C}_{(1,2)}^{\prime}(\zeta)=\left(2^{\widehat{m}+1}-1\right), \\
& \mathscr{C}_{(2,2)}^{\prime}(\zeta)=2 \times 2^{\widehat{m}}, \\
& \mathscr{C}_{(2,3)}^{\prime}(\zeta)=8\left(2^{\widehat{m}}-1\right), \\
& \mathscr{C}_{(3,4)}^{\prime}(\zeta)=6\left(2^{\widehat{m}}-1\right), \\
& \mathscr{C}_{(3,5)}^{\prime}(\zeta)=9 \times 2^{\widehat{m}}-10, \\
& \mathscr{C}_{(3,6)}^{\prime}(\zeta)=5\left(2 \times 2^{\widehat{m}}-3\right) .
\end{aligned}
$$

By putting above values in equation (7), we have

$$
\widehat{\mathbb{Z}}_{3}^{\mathbb{M}}(\zeta)=(16)^{\kappa}(2)^{2 \kappa-1}(5015306502144)^{\kappa-1}(15)^{9 \kappa-10}(18)^{5(2 \kappa-3)}
$$




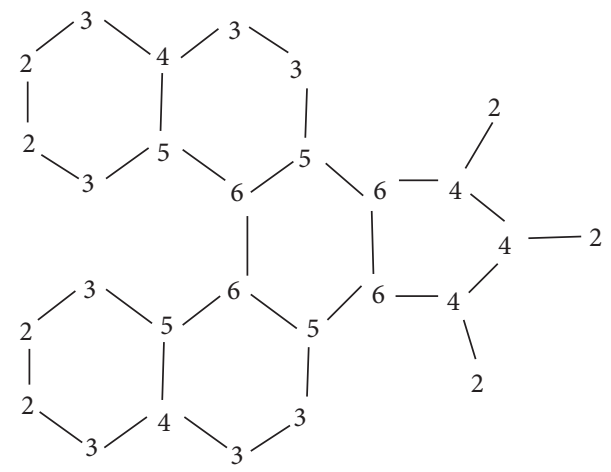

Figure $7: \mathbb{D}_{2}[1]$ along with the $\mathrm{CN}$ of each vertex.

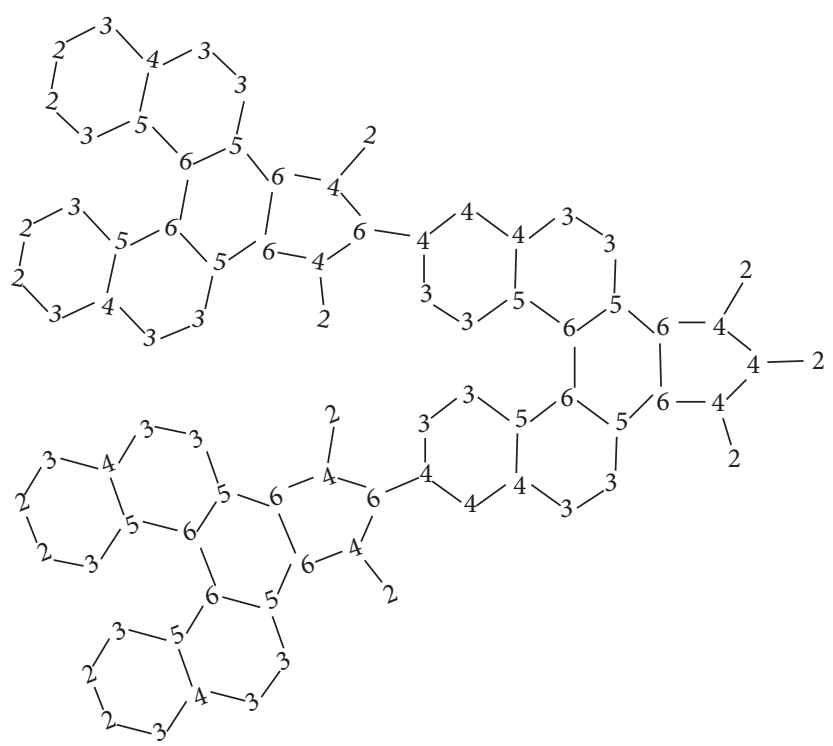

FIGURE 8: $\mathbb{D}_{2}[2]$ along with the $\mathrm{CN}$ of each vertex.

Theorem 4. Let $\zeta=\mathbb{N}_{2}[\hat{m}]$ be a molecular graph of order $\widehat{n}$. Then, second and fourth MZCIs of a molecular graph $\zeta$ are given as

(1) $\widehat{\mathbb{Z}}_{2}^{\mathbb{M}}(\zeta)=(148)^{\kappa}(8)^{2 \kappa}-1(9)^{3 \kappa-4}(24)^{5 \kappa-8}(180)^{4(\kappa-1)}$

(2) $\widehat{\mathbb{Z}}_{4}^{\mathbb{M}}(\zeta)=(100)^{\kappa}(6)^{2 \kappa-1}(6)^{3 \kappa-4}(10)^{5 \kappa-}$ $8(344534954841)^{\kappa-1}(37748736)^{\kappa-1}$, where $\kappa=2^{\hat{m}}$.

$$
\begin{aligned}
& \mathscr{C}_{(3,3)}(\zeta)=3 \times 2^{\widehat{m}}-4, \\
& \mathscr{C}_{(3,4)}(\zeta)=4\left(2^{\widehat{m}}-1\right), \\
& \mathscr{C}_{(3,5)}(\zeta)=4\left(2^{\widehat{m}}-1\right), \\
& \mathscr{C}_{(4,4)}(\zeta)=2\left(2^{\widehat{m}}-1\right), \\
& \mathscr{C}_{(4,5)}(\zeta)=2\left(2^{\widehat{m}}-1\right), \\
& \mathscr{C}_{(6,6)}(\zeta)=2\left(2^{\widehat{m}}-1\right), \\
& \mathscr{C}_{(4,6)}(\zeta)=5 \times 2^{\widehat{m}}-8, \\
& \mathscr{C}_{(5,6)}(\zeta)=6\left(2^{\widehat{m}}-1\right) .
\end{aligned}
$$

$$
\begin{aligned}
& \mathscr{C}_{(2,2)}(\zeta)=2^{\widehat{m}}, \\
& \mathscr{C}_{(2,3)}(\zeta)=2^{\widehat{m}+1}, \\
& \mathscr{C}_{(2,4)}(\zeta)=2^{\widehat{m}+1}-1,
\end{aligned}
$$




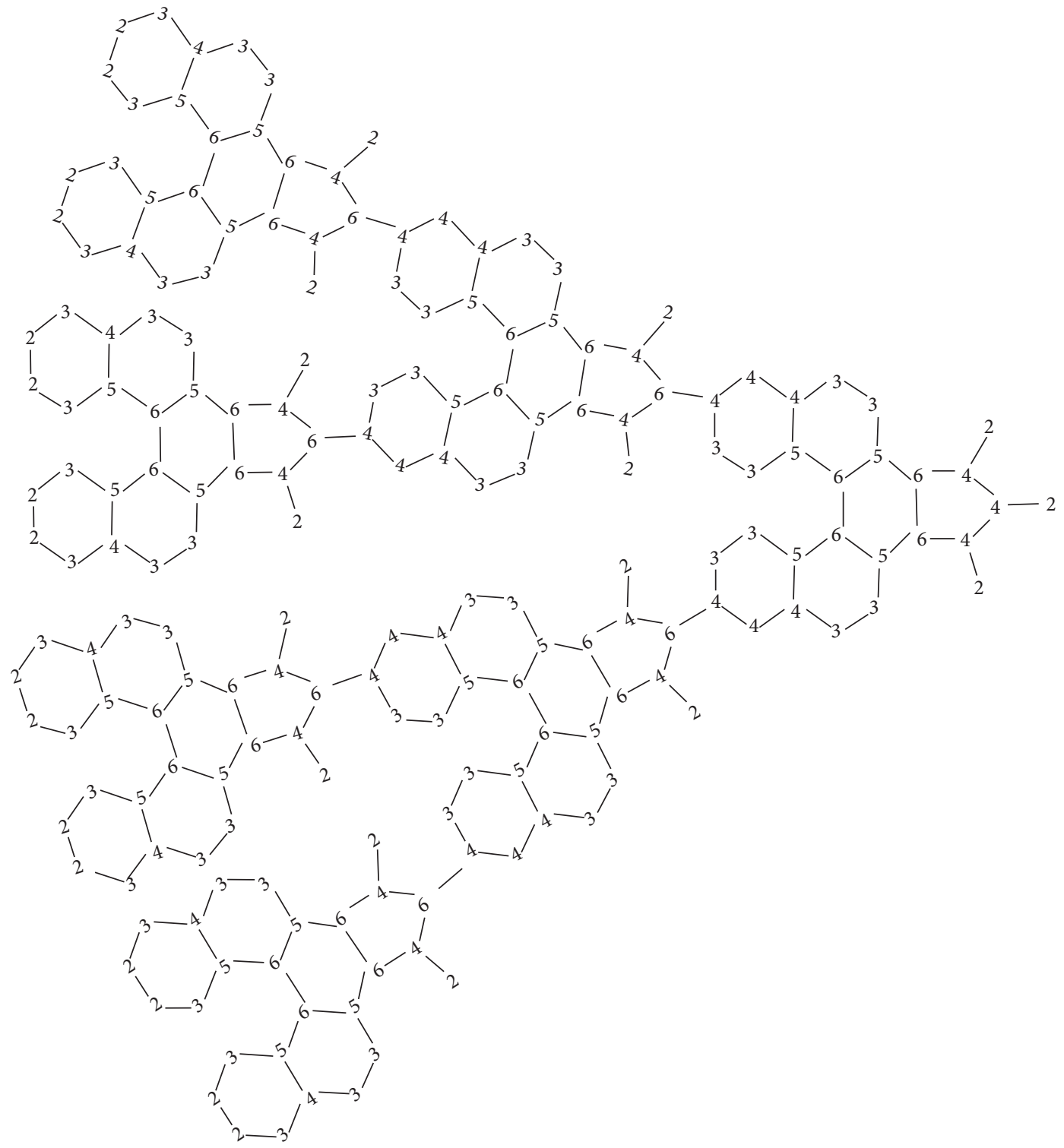

Figure 9: $\mathbb{D}_{2}[3]$ along with the $\mathrm{CN}$ of each vertex.

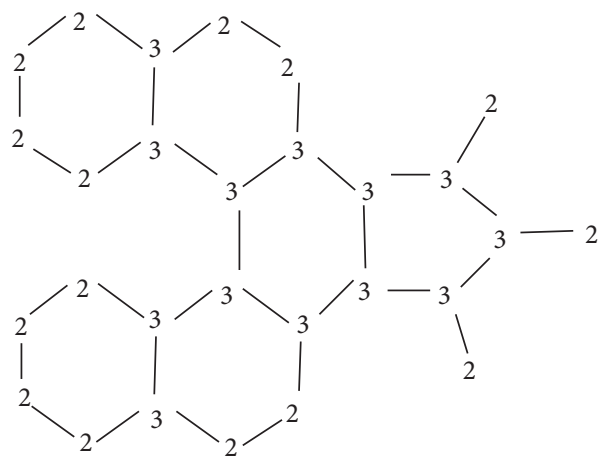

Figure 10: $\mathbb{D}_{2}[1]$ along with the degree of each vertex. 


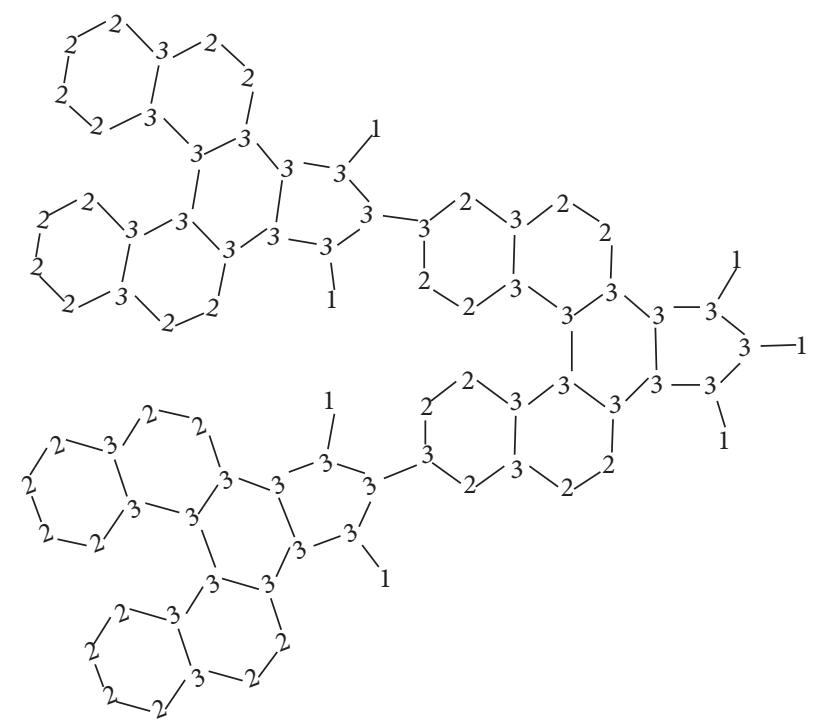

Figure 11: $\mathbb{D}_{2}[2]$ along with the degree of each vertex.

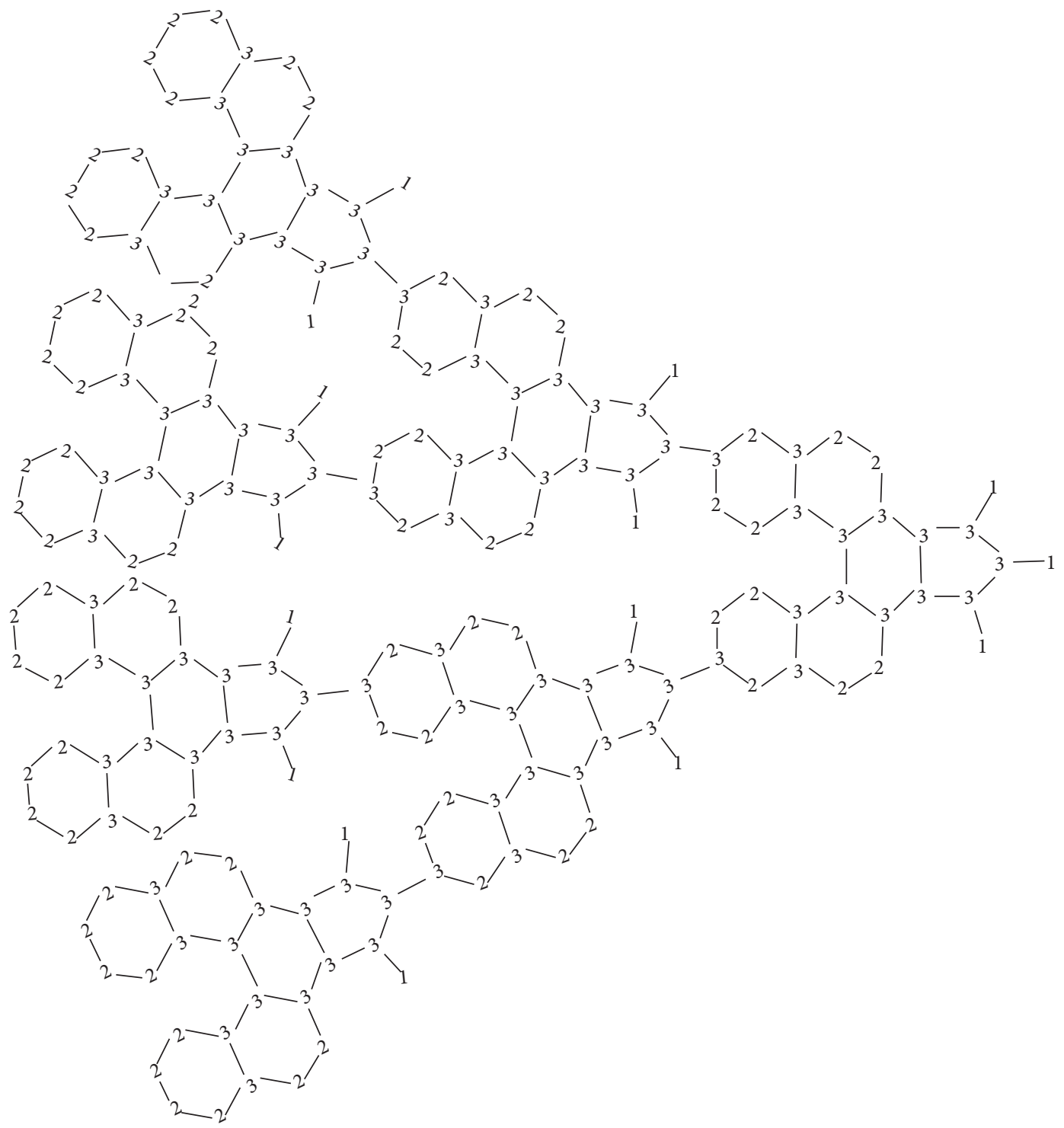

Figure 12: $\mathbb{D}_{2}[3]$ along with the degree of each vertex. 
TABLE 10: Comparison between the values of $\mathbb{D}_{1}[m]$ and $\mathbb{D}_{2}[m]$.

\begin{tabular}{lcc}
\hline ZMCIs & $\mathbb{D}_{1}[m]$ & $\mathbb{D}_{2}[m]$ \\
\hline$\widehat{\mathbb{Z}}_{\mathbb{M}}^{\mathbb{M}}(\zeta)$ & $(64)^{\kappa}(489776025600)^{\kappa-2}$ & $(4)^{4 \kappa-1}(16)^{6 \kappa-7}(16815125390625)^{\kappa-1}(36)^{5 \kappa-6}$ \\
$\widehat{\mathbb{Z}}_{\mathbb{R}}^{\mathbb{N}}(\zeta)$ & $(576)^{\kappa}(1990656000)^{(\kappa-2)}(81)^{2 \kappa-5}$ & $(148)^{\kappa}(8)^{2 \kappa-1}(9)^{3 \kappa-4}(24)^{5 \kappa-8}(180)^{4(\kappa-1)}(11520)^{2(\kappa-1)}(30)^{6(\kappa-1)}$ \\
$\widehat{\mathbb{Z}}_{\mathbb{R}}^{\mathbb{M}}(\zeta)$ & $(64)^{\kappa}(7255941120)^{(\kappa-2)}$ & $(16)^{\kappa}(2)^{2 \kappa-1}(5015306502144)^{\kappa-1}(15)^{9 \kappa-10}(18)^{5(2 \kappa-3)}$ \\
$\widehat{\mathbb{Z}}_{4}^{\mathbb{M}}(\zeta)$ & $(400)^{\kappa}(12252303)^{(\kappa-2)}(36)^{2 \kappa-5}$ & $(100)^{\kappa}(6)^{2 \kappa-1}(6)^{3 \kappa-4}(10)^{5 \kappa-8}(344534954841)^{\kappa-1}(37748736)^{\kappa-1}$ \\
\hline
\end{tabular}

By putting above values in equation (6), we have

$$
\widehat{\mathbb{Z}}_{2}^{\mathbb{M}}(\zeta)=(148)^{\kappa}(8)^{2 \kappa-1}(9)^{(3 \kappa-4)}(24)^{5 \kappa-8}(180)^{4(\kappa-1)}(11520)^{2(\kappa-1)}(30)^{6(\kappa-1)}
$$

(2) By putting all the values of $\mathscr{C}_{(\alpha, \beta)}$ in equation (8), we have the following expression:

$$
\widehat{\mathbb{Z}}_{4}^{\mathbb{M}}(\zeta)=(100)^{\kappa}(6)^{2 \kappa-1}(6)^{3 \kappa-4}(10)^{5 \kappa-8}(344534954841)^{\kappa-1}(37748736)^{\kappa-1}
$$

\section{Comparison Analysis}

In this section, to check the validity and superiority, we compare our calculated values for both the dendrimers with each other. Table 10 shows the comparison between the proposed results of dendrimers.

From Table 10, it can be seen that $\mathbb{D}_{1}[m]$ has the largest value for $\widehat{\mathbb{Z}}_{2}^{\mathbb{M}}(\zeta)$ while $\mathbb{D}_{2}[m]$ has the greatest value of $\widehat{\mathbb{Z}}_{2}^{\mathbb{M}}(\zeta)$.

\section{Conclusions}

Dendrimers are highly branched organic macromolecules with successive layers or generations of branch units surrounding a central core and are considered as key molecules in nanotechnology. In this research article, we have established the general expressions to find the connection-based MZIs, namely, first multiplicative Zagreb connection index, second multiplicative Zagreb connection index, third multiplicative Zagreb connection index, and fourth multiplicative Zagreb connection index of two well-known dendrimer nanostars. These calculated general expressions just depend upon the step of growth of these dendrimers. Moreover, to check the authenticity, we have also compared the calculated result for both types of dendrimers. In future, we are interested in extending our work for other types of dendrimers which are poly(propyl) ether imine dendrimer and polypropylenimine octamin dendrimer.

\section{Data Availability}

The data used to support the findings of this study are included within this article. However, the readers may contact the corresponding author for more details on the data.

\section{Conflicts of Interest}

The authors declare that they have no conflicts of interest.

\section{References}

[1] H. U. Afzal and T. Fatima, "On topological indices of OT [m, n] octagonal tillings and $\mathrm{TiO}_{2}$ nanotubes," Acta Chimica Slovenica, vol. 66, no. 2, pp. 435-442, 2019.

[2] A. Ali, I. Gutman, E. Milovanovic, and I. Milovanovic, "Sum of powers of the degrees of graphs extremal results and bounds," MATCH Communications in Mathematical and in Computer Chemistry, vol. 80, no. 1, pp. 5-84, 2018.

[3] U. Ali, M. Javaid, and A. Kashif, "Modified Zagreb connection indices of the T-sum graphs," Main Group Metal Chemistry, vol. 43, no. 1, pp. 43-55, 2020.

[4] A. Ali and N. Trinajstić, "A novel/old modification of the first Zagreb index," Molecular informatics, vol. 37, no. 6-7, Article ID e1800008, 2018.

[5] A. Ali, L. Zhong, and I. Gutman, "Harmonic index and its generalizations extremal results and bounds," $M A T C H$ Communications in Mathematical and in Computer Chemistry, vol. 81, no. 2, pp. 249-311, 2019.

[6] B. Borovicanin, K. C. Das, B. Furtula, and I. Gutman, "Bounds for zagreb indices," MATCH Communications in Mathematical and in Computer Chemistry, vol. 78, no. 1, pp. 17-100, 2017.

[7] S. A. U. H. Bokhary, M. Imran, and S. Manzoor, "On molecular topological properties of dendrimers," Canadian Journal of Chemistry, vol. 94, no. 2, pp. 120-125, 2016.

[8] J. Cao, U. Ali, M. Javaid, and C. Huang, "Zagreb connection indices of molecular graphs based on operations," Complexity, vol. 2020, Article ID 7385682, 15 pages, 2020.

[9] L. Chen, A. Mehboob, H. Ahmad, W. Nazeer, M. Hussain, and M. R. Farahani, "Hosoya and Harary polynomials of TOX(n),RTOX(n),TSL(n) and RTSL(n)," Discrete Dynamics in Nature and Society, vol. 2019, Article ID 8696982, 18 pages, 2019.

[10] Y. Chu, M. K. Shafiq, M. Imran et al., "The sharp bounds of Zagreb indices on connected graphs," Journal of Mathematical Inequalities, vol. 15, no. 2, pp. 477-489, 2021.

[11] K. C. Das, A. Yurttas, M. Togan, A. S. Cevik, and I. N. Cangul, "The multiplicative Zagreb indices of graph operations," 
Journal of Inequalities and Applications, vol. 2013, no. 1, pp. 1-14, 2013.

[12] K. Dhanalakshmi, J. Amalorpava Jerline, and L. Benedict Michaelraj, "Modified and multiplicative zagreb indices on graph operators," Journal of Computer and Mathematical Sciences, vol. 7, no. 4, pp. 225-232, 2016.

[13] N. Dorosti, A. Iranmanesh, and M. V. Diudea, "Computing the Cluj index of the first type dendrimer nanostar," Optoelectronics and Advanced Materials-Rapid Communications, vol. 4, pp. 381-384, 2010.

[14] J. Fang, I. Ahmed, A. Mehboob, K. Nazar, and H. Ahmad, "Irregularity of block shift networks and Hierarchical Hypercube networks," Journal of Chemistry, vol. 2019, Article ID 1042308, 12 pages, 2019.

[15] J. Fang, M. Rafiullah, and H. M. A. Siddiqui, "Topological properties of Sierpinski network and its application," Combinatorial Chemistry and High Throughput Screening, vol. 24, 2021.

[16] N. Fatima, A. A. Bhatti, A. ALi, and W. Gao, "Zagreb connection indices of two dendrimer nanostars," Acta Chemica Iasi, vol. 27, no. 1, pp. 1-14, 2019.

[17] B. Furtula and I. Gutman, "A forgotten topological index," Journal of Mathematical Chemistry, vol. 53, no. 4, pp. 11841190, 2015.

[18] W. Gao, M. Younas, A. Farooq, A. Mahboob, and W. Nazeer, "M-polynomials and degree-based topological indices of the crystallographic structure of molecules," Biomolecules, vol. 8, no. 4, p. 107, 2018.

[19] W. Gharibi, A. Ahmad, and M. K. Siddiqui, "On Zagreb indices, Zagreb polynomials of nanocone and nanotubes," Journal of Computational and Theoretical Nanoscience, vol. 13, no. 8, pp. 5086-5092, 2016.

[20] M. Ghorbani and N. Azimi, "Note on multiple Zagreb indices," Iranian Journal of Mathematical Chemistry, vol. 3, no. 2, pp. 137-143, 2012.

[21] I. Gutman, B. Ruscic, N. Trinajstic, and C. F. Wilcox Jr., "Graph theory and molecular orbitals. XII. Acyclic polyenes," The Journal of Chemical Physics, vol. 62, no. 9, pp. 3399-3405, 1975.

[22] I. Gutman and N. Trinajstić, "Graph theory and molecular orbitals. Total $\varphi$-electron energy of alternant hydrocarbons," Chemical Physics Letters, vol. 17, no. 4, pp. 535-538, 1972.

[23] J. Hao, "Theorems about Zagreb indices and modified Zagreb indices," MATCH Communications in Mathematical and in Computer Chemistry, vol. 65, pp. 659-670, 2011.

[24] R. S. Haoer, M. A. Mohammed, T. Selvarasan, N. Chidambaram, and N. Devadoss, "Multiplicative leap Zagreb indices of T-thorny graphs," Eurasian Chemical Communications, vol. 2, no. 8, pp. 841-846, 2020.

[25] M. Imran, A. Q. Baig, and H. Ali, "On molecular topological properties of hex-derived networks," Journal of Chemometrics, vol. 30, no. 3, pp. 121-129, 2016.

[26] A. Iqbal, G. Ali, J. Khan, M. N. G. Rahmat, and A. Kausar, "On topological indices of dual graph of benzene ring embedded in P-type surface in 2D network," International Journal, vol. 10, no. 3, 2021.

[27] M. Javaid, U. Ali, and J. B. Liu, "Computing analysis for first Zagreb connection index and coindex of resultant graphs," Mathematical Problems in Engineering, vol. 2021, Article ID 6019517, 19 pages, 2021.

[28] M. Javaid, U. Ali, and K. Siddiqui, "Novel connection based Zagreb indices of several wheel-related graphs," Computational Journal of Combinatorial Mathematics, vol. 1, pp. 1-28, 2021.
[29] P. Kesharwani, A. Gothwal, A. K. Iyer, K. Jain, M. K. Chourasia, and U. Gupta, "Dendrimer nanohybrid carrier systems: an expanding horizon for targeted drug and gene delivery," Drug Discovery Today, vol. 23, no. 2, pp. 300-314, 2018.

[30] M. H. Khalifeh, M. R. Darafsheh, and H. Jolany, "The Wiener, Szeged, and PI indices of a dendrimer nanostar," Journal of Computational and Theoretical Nanoscience, vol. 8, no. 2, pp. 220-223, 2011.

[31] B. Klajnert, L. Peng, and V. Ceña, Dendrimers in Biomedical Applications, Royal Society of Chemistry, London, UK, 2013.

[32] J. B. Liu, Z. Raza, and M. Javaid, "Zagreb connection numbers for cellular neural networks," Discrete Dynamics in Nature and Society, vol. 2020, Article ID 8038304, 8 pages, 2020.

[33] J. P. Mazorodze, S. Mukwembi, and T. Vetrík, "On the Gutman index and minimum degree," Discrete Applied Mathematics, vol. 173, pp. 77-82, 2014.

[34] S. Nikolic, G. Kovacevic, A. Milicevic, and N. Trinajstic, "The Zagreb indices 30 years after," Croatica Chemica Acta, vol. 76, no. 2, pp. 113-124, 2003.

[35] M. K. Siddiqui, M. Imran, and A. Ahmad, "On Zagreb indices, Zagreb polynomials of some nanostar dendrimers," Applied Mathematics and Computation, vol. 280, pp. 132-139, 2016.

[36] C. Rui, G. Ali, G. Rahmat, M. Y. Khan, A. SemanicovaFenovcikova, and J.-B. Liu, "Investigation of general power sum-connectivity index for some classes of extremal graphs," Complexity, vol. 2021, Article ID 6623277, 8 pages, 2021.

[37] J. H. Tang, U. Ali, M. Javaid, and K. Shabbir, "Zagreb connection indices of subdivision and semi-total point operations on graphs," Journal of Chemistry, vol. 2019, Article ID 9846913, 14 pages, 2019.

[38] R. Todeschini and V. Consonni, Molecular Descriptors for Chemoinformatics: Volume I: Alphabetical Listing/volume II: Appendices, John Wiley \& Sons, Hoboken, NJ, USA, 2009.

[39] H. Wiener, "Structural determination of paraffin boiling points," Journal of the American Chemical Society, vol. 69, no. 1, pp. 17-20, 1947. 